\title{
MULTIPLE PERIODIC SOLUTIONS FOR EIGENVALUE PROBLEMS WITH A $p$-LAPLACIAN AND NON-SMOOTH POTENTIAL
}

\author{
Guoqing Zhang and Sanyang LiU
}

\begin{abstract}
In this paper, we establish a multiple critical points theorem for a one-parameter family of non-smooth functionals. The obtained result is then exploited to prove a multiplicity result for a class of periodic eigenvalue problems driven by the $p$-Laplacian and with a non-smooth potential. Under suitable assumptions, we locate an open subinterval of the eigenvalue.
\end{abstract}

\section{Introduction}

In this paper, we consider the following periodic eigenvalue problems with a non-smooth potential (hemivariational inequality):

$$
\left\{\begin{array}{l}
-\left(\left|x^{\prime}(t)\right|^{p-2} x^{\prime}(t)\right)^{\prime} \in \lambda \partial j(t, x(t)) \quad \text { for almost all } t \in[0, b], \\
x(0)=x(b), \quad x^{\prime}(0)=x^{\prime}(b),
\end{array}\right.
$$

where $1<p<\infty, \lambda$ is a real parameter and $\partial j(x, t)$ is the generalized subdifferential of a generally non-smooth locally Lipschitz potential $\xi \rightarrow j(t, \xi)$.

Recently, there has been increasing interest in scalar periodic problems driven by the one dimensional $p$-Laplacian. We refer to the works for Fabry, Fayyad [6], Guo [10] and Dang, Oppenheimer [4]. However, in all these works, the approach is degree theory and in particular the fixed point index and Kransnoselski's theorem on fixed points for maps of the compression-expansion type. Moreover, they only establish the existence of one solution. In 1992, Del Pino, Manasevich, Murua [5] examined the problem of existence of multiple periodic solutions. Their approach used degree theory and assumed the forcing term $f(t, \xi)$ is continuous. Their conditions on $f(t, \xi)$ also require that

Received June 28, 2009; Revised July 25, 2009.

2010 Mathematics Subject Classification. 34C25.

Key words and phrases. multiple periodic solutions, critical points of non-smooth functionals, eigenvalue problems, $p$-Laplacian.

This research is supported by Innovation Program of Shanghai Municipal Education Commission under Grant 08YZ93 and Leading Academic Discipline Project of Shanghai Municipal Government, Project Number: S30501. 
asymptotically there is no interaction between the nonlinearity and the Fucik spectrum of the one dimensional $p$-Laplacian. In 2004, using smooth critical point theory and the so-called "second deformation theorem", E. H. Papageorgiou, N. S. Papageorgiou [14] established the existence of at least two nontrivial solutions.

The study of problems with non-smooth critical potential is lagging behind. In 2003, As $\lambda=1$, Gasinski, Papageorgiou [9] established three multiplicity results for Problem $(\mathrm{P})$ by using the non-smooth critical point theory of Chang [3] and its extensions due to Kourogenis, Papageorgiou [11]. In 2007, Gasinski [7] considered the following periodic system

$$
\left\{\begin{array}{l}
-\left(A\left(t, x^{\prime}(t)\right)^{\prime} \in \partial j(t, x(t)) \quad \text { for almost all } t \in[0, b],\right. \\
x(0)=x(b), \quad x^{\prime}(0)=x^{\prime}(b),
\end{array}\right.
$$

where $A:(0, b) \times \mathbb{R}^{N} \rightarrow 2^{\mathbb{R}^{N}}$ is a set valued map, and studied the existence of multiple solutions for problem (1.2) in both resonant and non-resonant cases by using an abstract local linking result.

In particular, in 2002, Marano, Motreanu [13] established a three critical points theorem, which is a non-smooth version of Ricceri's three critical points theorem. In 2005, using the non-smooth critical points theorem, Bonanno, Giovannelli [2] obtained a multiplicity result for an eigenvalue Dirichlet problem involving the $p$-Laplacian operator.

The aim of this paper is to prove a multiplicity result for Problem $(\mathrm{P})$. We establish a three critical points theorem and extended a three critical points theorem introduced by B. Ricceri to non-smooth functionals. It is worth pointing out that, we locate the interval of the parameter $\lambda$ and obtain the upper and lower bound of $\lambda$. As an application of the three critical points theorem, we obtain the existence of a bounded interval $\Lambda$, such that, for each $\lambda \in \Lambda$, Problem (P) admits three solutions.

We should mention that hemivariational inequalities arise in physical problems, when one wants to consider more realistic models with non-smooth and non-convex energy functionals. The hemivariational inequalities formalism proved to be an effective analytical tool in the study of many complex mechanical structures, such as multilayered plates, Vonkarman plates in adhesive contact with rigid support, composite structures, and others (see [8]). This paper is arranged as follows. In Section 2 we recall some basic definitions and establish a three critical points theorem, while Section 3 is devoted to multiplicity result for Problem (P).

\section{Three critical points theorem}

Let $X$ be a Banach space and $X^{*}$ be its topological dual. By \|\|$_{X}$, we denote the norm of $X$ and by $\langle,\rangle_{X}$ the duality brackets for the pair $\left(X, X^{*}\right)$. A functional $\Phi: X \rightarrow \mathbb{R}$ is called locally Lipschitz if for each $x \in X$, there exist 
a neighborhood $U$ of $x$ and a constant $L \geq 0$ such that

$$
\left|\Phi\left(x_{1}\right)-\Phi\left(x_{2}\right)\right| \leq L\left\|x_{1}-x_{2}\right\|_{X}, \quad \forall x_{1}, x_{2} \in U
$$

We define the generalized directional derivative of $\Phi$ at $x \in X$ along the direction $h \in X$, by

$$
\Phi^{0}(x ; h)=\limsup _{t \downarrow 0, x^{\prime} \rightarrow x} \frac{1}{t}\left[\Phi\left(x^{\prime}+t h\right)-\Phi(x)\right] .
$$

The generalized gradient of the functional $\Phi$ at $x$, denote by $\partial \Phi(x)$ is the set

$$
\partial \Phi(x)=\left\{x^{*} \in X^{*}:\left\langle x^{*}, h\right\rangle_{X} \leq \Phi^{0}(x ; h), \forall h \in X\right\} .
$$

It is obvious that if $\Phi \in C^{1}(X)$, then $\partial \Phi(x)=\left\{\Phi^{\prime}(X)\right\}, \forall x \in X$.

Definition 2.1. A point $x \in X$ is said to be a critical point of the locally Lipschitz functional $\Phi$ if $0 \in \partial \Phi(x)$, namely if one has $\Phi^{0}(x ; h) \geq 0$ for all $h \in X$.

Definition 2.2. A locally Lipschitz functional $\Phi$ satisfies the non-smooth (PS) condition if any sequence $\left\{x_{n}\right\} \subset X$ such that

$$
\Phi\left(x_{n}\right) \rightarrow c_{0}, c_{0} \in \mathbb{R} \text { and } \Phi^{0}\left(x_{n} ; h-x_{n}\right) \geq-\varepsilon_{n}\left\|h-x_{n}\right\|_{X},
$$

where $\varepsilon_{n} \rightarrow 0^{+}$, possesses a convergent subsequence.

Lemma 2.1 ([8]). Let $X$ be a reflexive Banach space and $\Phi: X \rightarrow \mathbb{R}$ a locally Lipschitz function with the non-smooth (PS) condition. If $\Phi$ has a pair of local minima (or maxima) $x_{0}, x_{1} \in X$, then $\Phi$ possesses at least three critical points.

Lemma $2.2([16])$. Let $X$ be a reflexive Banach space with norm \|\|$_{X}$, and let $M \subset X$ be a weakly closed subset of $X$. Suppose $E: M \rightarrow \mathbb{R} \cup\{+\infty\}$ is coercive and weak (sequentially) lower semi-continuous on $M$ with respect to $X$. That is

1) $E(x) \rightarrow \infty$ as $\|x\|_{X} \rightarrow \infty$;

2) For any sequence $\left\{x_{n}\right\}$ in $X$ such that $x_{n} \rightarrow x$ weakly as $n \rightarrow \infty$, there holds

$$
E(x) \leq \liminf _{n \rightarrow \infty} E\left(x_{n}\right) .
$$

Then $E$ is bounded from below on $M$ and attains a global minimum in $M$.

Now, we establish a multiple critical points theorems by using Lemma 2.1 and Lemma 2.2.

Theorem 2.3. Let $X$ be a separable reflexive real Banach space with norm \|\|$_{X}$, and let $\Phi_{1}, \Phi_{2}: X \rightarrow \mathbb{R}$ be two locally Lipschitz functions. Assume $\Phi_{1}$ is weakly sequentially lower semicontinuous and $\Phi_{2}$ is weakly continuous, there exists $r \in\left(\inf _{X} \Phi_{2}(x), \sup _{X} \Phi_{2}(x)\right)$ such that

$$
\varphi_{1}(r)<\varphi_{2}(r),
$$


where

$$
\left\{\begin{array}{l}
\varphi_{1}(r)=\inf \left\{\frac{\inf _{x \in \Phi_{2}^{-1}(r)} \Phi_{1}(x)-\Phi_{1}\left(x_{0}\right)}{\Phi_{2}\left(x_{0}\right)-r} \mid x_{0} \in \Phi_{2}^{-1}((-\infty, r))\right\}, \\
\varphi_{2}(r)=\sup \left\{\frac{\inf _{x \in \Phi_{2}^{-1}(r)} \Phi_{1}(x)-\Phi_{1}\left(x_{0}\right)}{\Phi_{2}\left(x_{0}\right)-r} \mid x_{0} \in \Phi_{2}^{-1}((r,+\infty))\right\} .
\end{array}\right.
$$

Further, we take a real interval $\Lambda$ and for $\lambda \in \Lambda$, the functional $\Phi_{1}+\lambda \Phi_{2}$ satisfies non-smooth (PS) condition and

$$
\lim _{\|x\|_{X} \rightarrow+\infty}\left(\Phi_{1}(x)+\lambda \Phi_{2}(x)\right)=+\infty .
$$

Then, in case $\left(\varphi_{1}(r), \varphi_{2}(r)\right) \cap \Lambda \neq \emptyset$, the functional $\Phi_{1}+\lambda \Phi_{2}$ admits at least three critical points for every $\lambda \in\left(\varphi_{1}(r), \varphi_{2}(r)\right) \cap \Lambda$.

Proof. Since $\Phi_{2}$ is weakly continuous, we obtain that the set $\Phi_{2}^{-1}((-\infty, r])$ is weakly closed. Let $M=\Phi_{2}^{-1}((-\infty, r])$. Since $\Phi_{1}$ is weakly sequentially lower semicontinuous and $\Phi_{1}+\lambda \Phi_{2}$ satisfies (2.6), we deduce that the restriction of $\Phi_{1}+\lambda \Phi_{2}$ to $M$ attains its infimum by using Lemma 2.2 . Now, let $x_{\lambda}$ be the infimum of the function $\Phi_{1}+\lambda \Phi_{2}$ in $M$. If $\Phi_{2}\left(x_{\lambda}\right)=r$, then

$$
\begin{aligned}
\inf _{\Phi_{2}^{-1}(r)} \Phi_{1}(x) & \leq \Phi_{1}\left(x_{\lambda}\right)=\Phi_{1}\left(x_{\lambda}\right)+\lambda\left(\Phi_{2}\left(x_{\lambda}\right)-r\right) \\
& \leq \Phi_{1}(x)+\lambda\left(\Phi_{2}(x)-r\right), \quad \forall x \in \Phi_{2}^{-1}((-\infty, r]) .
\end{aligned}
$$

Hence, we obtain that $\lambda \leq \varphi_{1}(r)$. However, from $\lambda \in\left(\varphi_{1}(r), \varphi_{2}(r)\right) \cap \Lambda$, we have $\lambda>\varphi_{1}(r)$. So we infer that $\Phi_{1}+\lambda \Phi_{2}$ admits a local minmum in for every $\lambda \in\left(\varphi_{1}(r),+\infty\right) \cap \Lambda$.

Similarly, we obtain that $\Phi_{1}+\lambda \Phi_{2}$ also admits a local minmum in $\Phi_{2}^{-1}((-\infty$, $r]$ ) for every $\lambda \in\left(-\infty, \varphi_{2}(r)\right) \cap \Lambda$. Thus, since $\varphi_{1}(r)<\varphi_{2}(r)$, we obtain the existence of two local minima for every $\lambda \in\left(\varphi_{1}(r), \varphi_{2}(r)\right) \cap \Lambda$. Taking into account that $\Phi_{1}+\lambda \Phi_{2}$ satisfies the non-smooth (PS) condition, we obtain that $\Phi_{1}+\lambda \Phi_{2}$ admits at least three critical points by Lemma 2.1 .

Remarks. 1) In Theorem 2.3, we locate the three critical point interval and establish the upper and lower bound of the eigenvalue $\lambda$. For differentiable functionals, Bonanno [1, Theorem B] locate the three critical point interval only in the case $\Lambda=[0,+\infty)$. Hence, our result improves the previous one given in [1].

2) By a simple calculation, we obtain that the hypothesis (3.1) is equivalent to the following: there exists $r \in\left(\inf _{x} \Phi_{2}(x), \sup _{X} \Phi_{2}(x)\right)$ and $x_{1}, x_{2}$ such that

$$
\begin{aligned}
& \Phi_{2}\left(x_{1}\right)<r<\Phi_{2}\left(x_{2}\right) \\
& \inf _{x \in \Phi_{2}^{-1}(r)} \Phi_{1}(x)>\frac{\left(\Phi_{2}\left(x_{2}\right)-r\right) \Phi_{1}\left(x_{1}\right)-\left(\Phi_{2}\left(x_{1}\right)-r\right) \Phi_{1}\left(x_{2}\right)}{\Phi_{2}\left(x_{2}\right)-\Phi_{2}\left(x_{1}\right)} .
\end{aligned}
$$




\section{Multiplicity result}

In this section, we prove a multiplicity result for Problem (P) by using Theorem 2.3.

Let the Sobolev space

$$
W_{\text {per }}^{1, p}\left([0, b]: \mathbb{R}^{N}\right)=\left\{x \in W^{1, p}\left([0, b]: \mathbb{R}^{N}\right): x(0)=x(b)\right\}
$$

with the norm

$$
\begin{aligned}
\|x(t)\| & =\left(\int_{0}^{T}|x(t)|^{p} d t+\int_{0}^{T}\left|x^{\prime}(t)\right|^{p} d t\right)^{\frac{1}{p}} \\
& =\left(\|x(t)\|_{p}^{p}+\left\|x^{\prime}(t)\right\|_{p}^{P}\right)^{\frac{1}{p}}
\end{aligned}
$$

where \|\|$_{p}$ denotes the norm of the space $L^{p}\left([0, b]: \mathbb{R}^{N}\right)$. By an eigenvalue $\lambda$ of the minus scalar $p$-Lapcian $x \mapsto\left(\left|x^{\prime}\right|^{p-2} x^{\prime}\right)^{\prime}$ with $b$-periodic boundary condition, we mean a $\lambda \in \mathbb{R}$ such that the problem

$$
\left\{\begin{array}{l}
-\left(\left|x^{\prime}(t)\right|^{p-2} x^{\prime}(t)\right)^{\prime}=\lambda|x(t)|^{p-2} x(t) \quad \text { for almost all } t \in[0, T], \\
x(0)=x(T), x^{\prime}(0)=x^{\prime}(T)
\end{array}\right.
$$

has a non-trivial solution $x$, called the eigenfunction corresponding to $\lambda$. Evidently, $\lambda_{0}=0$ is an eigenvalue of (3.1) and is simple and isolated. Each eigenvalue is non-negative and 0 is the smallest one (see Mawhin [12]). So, if

$$
\lambda_{1}=\inf \left\{\lambda>0: \lambda \text { is an eigenvalue of }-\triangle_{p}\right\},
$$

then $\lambda_{1}>0$.

Assume $j:(-0, b) \times \mathbb{R}^{N} \rightarrow \mathbb{R}$ is a function such that

(H1) for all $\xi \in \mathbb{R}$, the function $\xi \rightarrow j(t, \xi)$ is measurable;

(H2) for almost all $t \in[0, b]$, the function $\xi \rightarrow j(t, \xi)$ is locally Lipschitz and $j(t, 0)=0$;

(H3) for almost all $t \in[0, b]$ and all $u \in \partial j(t, \xi)$, we have

$$
|u| \leq c_{1}(t)+c_{2}(t)|\xi|^{r-1},
$$

with $c_{1}, c_{2} \in L^{r^{\prime}}((0, b))_{+}$, where $1 \leq r<+\infty$ and $\frac{1}{r}+\frac{1}{r^{\prime}}=1$;

(H4) $\lim \sup _{|\xi| \rightarrow+\infty} \frac{p j(t, \xi)}{|\xi|^{p}}<0$ uniformly for almost all $t \in[0, b]$;

(H5) for some $\gamma>p$, we have

$$
\limsup _{|\xi| \rightarrow 0} \frac{|j(t, \xi)|}{|\xi|^{\gamma}}<+\infty .
$$

Now, we define the functionals $\Phi_{1}, \Phi_{2}: W_{\mathrm{per}}^{1, p}([0, b]) \rightarrow \mathbb{R}$ by

$$
\Phi_{1}(x(t))=\frac{1}{p}\left\|x^{\prime}\right\|_{p}^{p}, \quad \Phi_{2}(x(t))=-\int_{0}^{b} j(t, x(t)) d t,
$$

and the function $\varphi_{1}, \varphi_{2}$ given respectively by (2.6). 
Theorem 3.1. Assume (H1)-(H5) be satisfied and $j^{+}(t, \xi) \neq 0$, then for each compact interval $\Lambda \subset\left(\varphi_{1}(0),+\infty\right)$, Problem $(\mathrm{P})$ admits at least two non-trivial solution for every $\lambda \in \Lambda$.

Remark. By (H2), we obtain that $x=0$ is a trivial solution of Problem $(\mathrm{P})$.

Let the functional $I: W_{\text {per }}^{1, p}([0, b]) \rightarrow \mathbb{R}$ by

$$
\begin{aligned}
I(x) & =\frac{1}{p}\left\|x^{\prime}\right\|_{p}^{p}-\lambda \int_{0}^{b} j(t, x(t)) d t \\
& =\Phi_{1}(x)+\lambda_{2}(x) .
\end{aligned}
$$

Lemma 3.2. The functional $I(x)$ is locally Lipschitz in $W_{\mathrm{per}}^{1, p}([0, b])$.

Proof. By (H2), (H3) and Lebourg mean value theorem, we obtain a $u_{0} \in$ $\partial j(t, \xi)$ such that

$$
\begin{aligned}
\mid j(t, \xi \mid & =|j(t, \xi)-j(t, 0)| \leq u_{0} \xi \\
& =c_{3}(t)+c_{4}(t)|\xi|^{r},
\end{aligned}
$$

with $c_{3} \in L^{1}([0, b]), c_{4} \in L^{r^{\prime}}([0, b])$. By (3.3) and a standard argument in Chang $([3])$, we can obtain that $\Phi_{2}(x)=-\int_{0}^{b} j(t, x(t)) d t$ is locally Lipschitz continuous. Since $\Phi_{1}(x)=\frac{1}{p}\left\|x^{\prime}\right\|_{p}^{p}$ is of class $C^{1}$ and $\lambda$ is a real parameter, we obtain that $I(x)$ is locally Lipschitz in $W_{\text {per }}^{1, p}([0, b])$.

By standard results, the functional $\Phi_{1}(x)=\frac{1}{p}\left\|x^{\prime}\right\|_{p}^{p}$ is weakly sequentially lower semicontinuous. Since (H3) holds and the compactness of the embedding $W_{\text {per }}^{1, p}([0, b]) \subset C([0, b])$, we can obtain that $\Phi_{2}(x)=-\int_{0}^{b} j(t, x(t)) d t$ is also weakly (upper semi)continuous and non-constant.

Lemma 3.3. Every critical $x \in W_{\mathrm{per}}^{1, p}([0, b])$ of the functional $I(x)$ is a weak solution of Problem (P).

Proof. Let $x \in W_{\text {per }}^{1, p}([0, b])$ be a critical point of the functional $I(x)$. Then we have $0 \in \partial I(x)$ and so we can find $u \in S_{\partial j(\cdot, x(\cdot))}^{r^{\prime}}$ such that

$$
\left.\int_{0}^{b}\left|x^{\prime}(t)\right|^{p-2} x^{\prime}(t)\right)^{\prime} \theta^{\prime}(t) d t=\lambda \int_{0}^{b} u(t) \theta(t) d t, \quad \forall \theta \in C_{0}^{\infty}([0, b]),
$$

where $S_{\partial j(\cdot, x(\cdot))}^{r^{\prime}}=\left\{\nu \in L^{r^{\prime}}([0, b]): \nu(t) \in \partial j(t, x(t))\right.$ a.e. on $\left.[0, b]\right\}$. Since $C_{0}^{\infty}([0, b])$ is dense in $W_{0}^{1, p}([0, b])$, we obtain that

$$
-\left(\left|x^{\prime}(t)\right|^{p-2} x^{\prime}(t)\right)^{\prime}=\lambda u(t) \in \lambda \partial j(t, x(t)) \text { a.e. on }[0, b] \text {. }
$$

From Green's identity, for every $\vartheta \in W_{\text {per }}^{1, p}([0, b])$ we have

$$
\begin{aligned}
& \lambda \int_{0}^{b} u(t) \vartheta(t) d t+\int_{0}^{b}\left(\left|x^{\prime}(t)\right|^{p-2} x^{\prime}(t)\right)^{\prime} \vartheta(t) d t \\
= & \left|x^{\prime}(b)\right|^{p-2} X^{\prime}(b) \vartheta(b)-\left|x^{\prime}(0)\right|^{p-2} x^{\prime}(0) \vartheta(0),
\end{aligned}
$$


and so by (3.4) we have

$$
\left|x^{\prime}(b)\right|^{p-2} X^{\prime}(b) \vartheta(b)=\left|x^{\prime}(0)\right|^{p-2} x^{\prime}(0) \vartheta(0) .
$$

Let $\vartheta \in W_{\text {per }}^{1, p}([0, b])$ be such that $\vartheta(b)=\vartheta(0)=1$. Then we obtain

$$
\left|x^{\prime}(b)\right|^{p-2} X^{\prime}(b) \vartheta(b)=\left|x^{\prime}(0)\right|^{p-2} x^{\prime}(0),
$$

and so

$$
x^{\prime}(b)=x^{\prime}(0),
$$

i.e., $x \in W_{\text {per }}^{1, p}([0, b])$ is a weak solution of Problem $(\mathrm{P})$.

Lemma 3.4. The functional is coercive and satisfies non-smooth (PS) condition.

Proof. By (H4), we obtain that there exist $R>0$ and $\alpha>0$ such that for almost all $t \in(0, T)$ and $|\xi| \geq R$

$$
p j(t, \xi) \leq-\alpha|\xi|^{p} .
$$

Combining (3.3) and (3.5), we obtain that for almost all $t \in(0, T)$ and $|\xi| \leq R$

$$
|j(t, \xi)| \leq c_{5}(t),
$$

with $c_{5} \in L^{1}([0, b])$. Therefore, we obtain that for any $\xi \in \mathbb{R}$,

$$
j(t, \xi) \leq-\frac{\alpha}{p}|\xi|^{p}+c_{6}(t)
$$

with $c_{6} \in L^{1}([0, b])$. So for every $x \in W_{\text {per }}^{1, p}([0, b))$, we obtain that

$$
\begin{aligned}
I(x)=\Phi_{1}(x)+\lambda \Phi_{2}(x) & \geq \frac{1}{p}\left\|x^{\prime}\right\|_{p}^{p}+|\lambda|\left(\frac{\alpha}{p}\|x\|_{p}^{p}-\alpha_{1}\right) \\
& \geq \frac{1}{p} \min \{1, \alpha\}\left(\left\|x^{\prime}\right\|_{p}^{p}+|\lambda|\|x\|_{p}^{p}\right)-\alpha_{1},
\end{aligned}
$$

with $\alpha_{1}>0$. This implies that the functional $I(x)$ is coercive for every $\lambda \in$ $\left(\varphi_{1}(0),+\infty\right)$. Therefore, $I(x)$ is bounded below, and hence it satisfies the nonsmooth (PS) condition.

The proof of Theorem 3.1. To apply Theorem 2.3, we only obtain that there exists $r \in \Phi_{2}(x(t))$ such that $\varphi_{1}(r)<\varphi_{2}(r)$. Indeed, it is easy to obtain that if $j^{+}(t, x(t))=0$, then $\Phi_{2}\left(x(t) \subset \mathbb{R} \cup\{0\}\right.$. If $j^{+}(t, x(t)) \neq 0$, there exists $x_{0}(t)$ such that $j^{+}(t, x(t))>0$. Given $\varepsilon>0$, we choose an open interval $T_{\varepsilon} \subset(0, b)$ with $\left|(0, b) \backslash T_{\varepsilon}\right|<\varepsilon$ and $x_{\varepsilon}(t)$ with compact support in $(0, b)$ such that $x_{\varepsilon}(t)=x_{0}(t)$ in $T_{\varepsilon}$ and $\left\|x_{\varepsilon}(t)\right\|_{\infty}=\left|x_{0}(t)\right|$. Therefore, we have

$$
\begin{aligned}
\Phi_{2}\left(x_{\varepsilon}(t)\right) & =-\int_{0}^{b} j\left(t, x_{\varepsilon}(t)\right) d t \\
& =-\int_{T_{\varepsilon}} j\left(t, x_{\varepsilon}(t)\right) d t-\int_{(0, b) \backslash T_{\varepsilon}} j\left(t, x_{\varepsilon}(t)\right) d t \\
& \leq-j\left(t, x_{0}(t)\right)\left|T_{\varepsilon}\right|+\varepsilon \max _{\left|x_{\varepsilon}(t)\right| \leq x_{0}(t)} j\left(t, x_{\varepsilon}(t)\right) .
\end{aligned}
$$


Hence, let $\varepsilon \rightarrow 0$, we have

$$
\limsup _{\varepsilon \rightarrow 0^{+}} \Phi_{2}\left(x_{\varepsilon}(t)\right) \leq-j\left(t, x_{0}(t)\right) b<0 .
$$

So we obtain that $\Phi_{2}(x(t))$ can take negative values and $\Phi_{2}^{-1}(-\infty, 0) \neq \emptyset$. For every $x(t) \in \Phi_{2}^{-1}(-\infty, 0)$, we have

$\varphi_{1}(r) \leq \frac{\inf _{x_{0} \in \Phi_{2}^{-1}(r)} \Phi_{1}\left(x_{0}(t)\right)-\Phi_{1}(x(t))}{\Phi_{2}(x(t))-r} \leq-\frac{\Phi_{1}(x(t))}{\Phi_{2}(x(t))-r}, \forall r \in\left(\Phi_{2}(x(t)), 0\right)$.

This implies that

$$
\limsup _{r \rightarrow 0^{-}} \varphi_{1}(r) \leq-\frac{\Phi_{1}(x(t))}{\Phi_{2}(x(t))}, \forall x(t) \in \Phi_{2}^{-1}(-\infty, 0)
$$

or equivalently

$$
\limsup _{r \rightarrow 0^{-}} \varphi_{1}(r) \leq \varphi_{1}(0) .
$$

On the other hand, by (H5) and (3.3), we obtain

$$
\left|\Phi_{2}(x(t))\right|=\left|-\int_{0}^{b} j(t, x(t)) d t\right| \leq c_{6}|x(t)|_{\gamma}^{\gamma} \leq c_{7}\left|x^{\prime}(t)\right|_{\gamma}^{\gamma}
$$

with $c_{6}, c_{7}>o$. Hence, there exists $r<0$ such that $x(t) \in \Phi_{2}^{-1}(r)$, we obtain that

$$
-r=-\Phi_{2}(x(t)) \leq c_{8}\left|x^{\prime}(t)\right|_{\gamma}^{\gamma},
$$

with $c_{8}>0$. So we have

$$
\begin{aligned}
\varphi_{2}(r) & \geq \frac{\inf _{x(t) \in \Phi_{2}^{-1}(r)} \Phi_{1}(x(t))-\Phi_{1}(x(0))}{\Phi_{2}(0)-r} \\
& =\frac{1}{p} \frac{\inf _{x(t) \in \Phi_{2}^{-1}(r)}\left|x^{\prime}(t)\right|_{p}^{p}}{-r} \geq c_{9} \frac{(-r)^{\frac{p}{\gamma}}}{-r},
\end{aligned}
$$

with $c_{9}>0$. Therefore, this implies that

$$
\lim _{r \rightarrow 0^{-}} \varphi_{2}(r)=+\infty \text {. }
$$

Combining (3.7) and (3.8), we can choose $r<0$ such that for each compact interval $\Lambda \subset\left(\varphi_{1}(0),+\infty\right)$

$$
\varphi_{1}(r)<\inf \Lambda \leq \sup \Lambda<\varphi_{2}(r) .
$$

The proof is concluded. 


\section{References}

[1] G. Bonanno, Some remarks on a three critical points theorem, Nonlinear Anal. 54 (2003), no. 4, 651-665.

[2] G. Bonanno and N. Giovannelli, An eigenvalue Dirichlet problem involving the $p$ Laplacian with discontinuous nonlinearities, J. Math. Anal. Appl. 308 (2005), no. 2, 596-604.

[3] K.-C. Chang, Variational methods for nondifferentiable functionals and their applications to partial differential equations, J. Math. Anal. Appl. 80 (1981), no. 1, 102-129.

[4] H. Dang and S. F. Oppenheimer, Existence and uniqueness results for some nonlinear boundary value problems, J. Math. Anal. Appl. 198 (1996), no. 1, 35-48.

[5] M. A. del Pino, R. Manásevich, and A. Murúa, Existence and multiplicity of solutions with prescribed period for a second order quasilinear ODE, Nonlinear Anal. 18 (1992), no. 1, 79-92.

[6] C. Fabry and D. Fayyad, Periodic solutions of second order differential equations with a p-Laplacian and asymmetric nonlinearities, Rend. Istit. Mat. Univ. Trieste 24 (1992), no. 1-2, 207-227.

[7] L. Gasinski, Multiplicity theorems for periodic systems with a p-Laplacian-like operator, Nonlinear Anal. 67 (2007), no. 9, 2632-2641.

[8] L. Gasinski and N. S. Papageorgiou, Nonsmooth Critical Point Theory and Nonlinear Boundary Value Problems, Chapman and Hall, CRC, Boca Raton, 2005.

[9] - On the existence of multiple periodic solutions for equations driven by the $p$ Laplacian and with a non-smooth potential, Proc. Edinb. Math. Soc. (2) 46 (2003), no. $1,229-249$.

[10] Z. M. Guo, Boundary value problems of a class of quasilinear ordinary differential equations, Differential Integral Equations 6 (1993), no. 3, 705-719.

[11] N. Kourogenis and N. S. Papageorgiou, Nonsmooth critical point theory and nonlinear elliptic equations at resonance, Kodai Math. J. 23 (2000), no. 1, 108-135.

[12] J. Mawhin, Periodic solutions of systems with p-Laplacian-like operators, Nonlinear analysis and its applications to differential equations (Lisbon, 1998), 37-63, Progr. Nonlinear Differential Equations Appl., 43, Birkhauser Boston, Boston, MA, 2001.

[13] S. A. Marano and D. Motreanu, On a three critical points theorem for non-differentiable functions and applications to nonlinear boundary value problems, Nonlinear Anal. 48 (2002), no. 1, Ser. A: Theory Methods, 37-52.

[14] E. H. Papageorgiou and N. S. Papageorgiou, Two nontrivial solutions for quasilinear periodic equations, Proc. Amer. Math. Soc. 132 (2004), no. 2, 429-434

[15] B. Ricceri, On a three critical points theorem, Arch. Math. (Basel) 75 (2000), no. 3, 220-226.

[16] M. Struwe, Varaitional Methods, Springer-Verlag, Berlin, 1996.

GUOQING ZHANG

College of SCIENCES

University of Shanghai for Science and Technology

Shanghai 200093, P. R. China

E-mail address: shzhangguoqing@126.com

SANYANG LIU

College of Sciences

Xidian University

XI'An 710071 ShaAnXi, P. R. China

E-mail address: liusanyang@126.com 